\title{
Function of miR-24 and miR-27 in Pediatric Patients With Idiopathic Nephrotic Syndrome
}

\section{Fen-fen Ni}

Shenzhen Children's Hospital

Guang-lei Liu

Affiliated Hospital of Zunyi Medical College

shi-lei Jia

Shenzhen Children's Hospital

\section{Ran-ran Chen}

Shenzhen Children's Hospital

\section{Li-bing Liu}

Shenzhen Children's Hospital

Cheng-rong Li ( $\square$ chengrongli0755@163.com )

Shenzhen Children's Hospital

Jun Yang

Shenzhen Children's Hospital

Xiao-jie Gao

Shenzhen Children's Hospital

\section{Research Article}

Keywords: Idiopathic nephrotic syndrome, miR-24, miR-27, Th2 cells, IL-4, IL-13

Posted Date: February 1st, 2021

DOI: https://doi.org/10.21203/rs.3.rs-150431/v1

License: (c) (1) This work is licensed under a Creative Commons Attribution 4.0 International License.

Read Full License 


\section{Function of miR-24 and miR-27 in pediatric patients with idiopathic nephrotic syndrome}

Running title: miRNAs in idiopathic nephrotic syndrome

List of authors: Fen-fen $\mathrm{Ni}^{1}$,Guang-lei Liu${ }^{2}$, Shi-lei Jia ${ }^{1}$, Ran-ran Chen ${ }^{1}$, Li-bing Liu ${ }^{1}$, Cheng-rong Li ${ }^{1 *}$, Jun Yang ${ }^{3 *}$, Xiao-Jie Gao ${ }^{1 *}$

Author's institutional affiliations: ${ }^{1}$ Department of Nephrology, Shenzhen

Children's Hospital, Shenzhen, 518026, China; ${ }^{2}$ The Fifth Affiliated (Zhuhai)

Hospital of Zunyi Medical University, Zhuhai, 519100, China; ${ }^{3}$ Department of

Immunology, Shenzhen Children's Hospital, Shenzhen, 518026, China.

Corresponding authors: Cheng-rong Li, Department of Nephrology, Shenzhen Children’s Hospital, Shenzhen, 518026, China; Email: chengrongli0755@163.com

Jun Yang, Department of Immunology, Shenzhen Children's Hospital, Shenzhen, 518026, China; shenzhen81111@163.com

Xiao-Jie Gao, Department of Nephrology, Shenzhen Children's Hospital, Shenzhen,

518026, China; Email: gxj0824@hotmail.com

* Co-Corresponding authors 


\begin{abstract}
Background: The specific etiology and mechanism of idiopathic nephrotic syndrome (INS) in children remain unclear, so we investigated the pathogenesis of INS by measuring the effects two specific miRNAs on Th2 cells in children with this disease.
\end{abstract}

Methods: Flow cytometry was used to measure the levels of Th2 cells and a cytometric bead array was used to measure the levels of IgE, interleukin (IL) -4, and IL-13. RT-PCR was used to measure the levels of miR-24 and miR-27 in CD4 ${ }^{+} \mathrm{TCD}^{2} 5^{-}$cells. PBMCs were isolated using Ficoll density gradient centrifugation, and transfected with different mimic or inhibitor miRNAs. RT-PCR was used to measure the expression of different RNAs, and flow cytometry was used to determine the percentages of $\mathrm{Th} 2$ cells.

Results: Children with active INS had higher percentages of Th2 cells than healthy controls $(\mathrm{P}<0.05)$, but there was no significant difference for children in remission. The plasma levels of IgE, IL-4, and IL-13 were significantly increased in children with active INS $(\mathrm{P}<0.05)$. miR-24 and miR-27 were at lower levels in children with active non-atopic INS $(\mathrm{P}<0.05)$. Transfection experiments indicated that upregulation of each miRNA decreased the percentage of Th2 cells and the level of IL-4 $(\mathrm{P}<0.05)$, and down-regulation of each miRNA had the opposite effects $(\mathrm{P}<0.05)$.

Conclusion: Children with active INS, with or without atopy, had higher levels of IgE, possibly related to their higher levels of IL-13 and IL-4 due to drift toward Th2 
cells. miR-24 and miR-27 suppress the expression of Th2 cells and have a critical function in Th2 expression in INS.

Keywords: Idiopathic nephrotic syndrome, miR-24, miR-27, Th2 cells, IL-4, IL-13

\subsection{Introduction}

Idiopathic nephrotic syndrome (INS) is the most common renal disease in children, and a main cause of chronic renal failure in children from China [1]. The clinical manifestations of INS are proteinuria, low level of plasma albumin, hyperlipidemia, and edema. The prevalence is greatest in preschool children who are 3 to 5 years-old. About 80 to $90 \%$ of patients with kidney disease who are under 10 years-old have minimal change disease (MCD), a common cause of INS [2]. However, the specific etiology and mechanism of INS in children remain unclear. Previous studies showed that INS is associated with immune system dysfunctions, including humoral immune disorders, T cell subset dysfunction, and abnormal secretion of cytokines, especially due to T cell dysfunction [3-8].

T helper type 2 (Th2) cells, which mainly produce IL-4, IL-5, and IL-13, play an major role in responses to parasitic infections and allergic inflammatory diseases [9-11]. A seminal 1959 study reported an association of pollen sensitivity with seasonal proteinuria, and that about $30 \%$ of children with INS had atopic 
manifestations, such as allergic rhinitis and idiopathic dermatitis [12]. Subsequent studies confirmed elevated levels of immunoglobulin E ( $\mathrm{IgE}$ ) during the active phase of INS [13-15], but it is unknown whether the increased levels of $\operatorname{IgE}$ in these children are pathogenic or coincidental.

We proposed a "two hit" hypothesis for the pathogenesis of INS. The first hit occurs when microbial products, allergens, or T-cell cytokines (such as IL-13) damage the glomeruli, resulting in the overexpression of CD80 by podocytes and temporary proteinuria [16-18]. In normal settings, regulatory cytokines produced by $\mathrm{T}$ regulatory cells (Tregs) terminate the CD80 overexpression, so that the proteinuria is transient and mild [18-20]. However, the second hit occurs when MCD is present, and this leads to a failure to block CD80 expression by podocytes due to a disruption of autoregulatory responses in Tregs or even in the podocytes themselves. After this second hit, CD80 expression remains continuously elevated, leading to nephrotic syndrome [20]. Th2 related cytokines such as IL-13, which stimulates IgE-mediated responses, can promote proteinuria in patients with MCD because it can directly induce CD80 expression in podocytes [13-15, 18]. Recent studies found increased levels of IL-13 and IL-4 during the active phase of INS, and that IL-13 functions in the pathogenesis of kidney disease $[13-15,18]$, but the mechanisms responsible for the increased level of IL-13 are still unclear. A few studies also reported that Th2 cells were over-active in INS [8,18, 21-22], but the mechanisms leading to over-activation of these cells are also unknown. Studies of these topics may help to elucidate the role of altered immune responses in the pathogenesis of INS. 
MicroRNAs (miRNAs) are short (20-23 nucleotides) non-coding RNAs that can alter target gene expression [23-24]. In particular, a specific miRNA binds to the 3'-untranslated region (3'-UTR) of its target mRNA, and this is followed by inhibition of translation or increased mRNA degradation [23-24]. Initially, most studies of miRNAs were in the field of oncology, but recently studies have examined their role in kidney disease. For example, several studies showed that multiple miRNAs can inhibit the differentiation and function of Th2 cells [25-27]. In 2016, two studies reported that miR-24 and miR-27 acted together via different downstream pathways to inhibit the differentiation of Th2 cells and block the synthesis of IL-4 [26-27]. However, the effects of these two miRNAs on Th2 expression in patients with active non-atopic INS, and their specific mechanisms need further study.

We systematically determined the dynamics of cytokines, miR-24, and miR-27 in the regulation of $\mathrm{Th} 2$ cell differentiation during the active and remission phases of INS, and measured changes in the number and function of Th2 cells isolated from children with non-atopic INS under conditions of altered miRNA expression. Our general purpose was to clarify the role of immune system alterations in the pathogenesis of INS, and to identify potential therapeutic targets and new ideas for the treatment of kidney disease.

\subsection{Materials and Methods}

\subsection{Subjects}


Forty children with INS (23 males and 17 females; median age: 38 months; age range: 22-92 months) were enrolled. All children were patients at Shenzhen Children's Hospital from September 2015 to October 2016. The inclusion criteria were: diagnosis of INS based on 2010 criteria determined using evidence-based diagnosis and treatment guidelines for common kidney diseases in children from China [28]; hormone-sensitivity, that is negative urinary protein within 4 weeks of treatment but with sufficient hormones and no use of other immunosuppressants; no secondary kidney disease (secondary nephrotic syndrome, nephrotic nephrotic syndrome or congenital kidney disease, etc.); no other systemic visceral syndrome; and signed informed consent by the parents or legal guardians.

The 40 children were divided into four groups: an active phase (first-onset) group with atopic constitution (AA, $\mathrm{n}=6)$, an active phase group with non-atopic constitution (ANA, $n=14)$, a remission group with atopic constitution $(\operatorname{Re} A, n=6)$, and a remission group with non-atopic constitution (ReNA, $n=14) .12$ Atopy was diagnosed on the basis of positive family history, clinical varieties of atopy (including asthma, recurrent urticarial, eczema, and allergic rhinitis), and elevated serum IgE concentration. The results of the skin-prick tests were positive for all the atopic children. Children in the INS group were recently diagnosed with INS and were not yet under treatment; All children in the remission groups had kidney disease, stopped using hormones for more than 4 weeks, and had normal blood biochemical and urine tests. In addition, 20 healthy children of similar age (11 males and 9 females; median age: 31.3 months; age range: $25-105$ months) who came to our hospital for physical 
examinations during the same period were enrolled as controls (Ctrl). The parents or legal guardians provided informed consent prior to study enrollment, and the study was performed after approval by the local Medical Ethics Committee.

\subsection{Blood samples}

Venous blood was collected from patients and healthy controls in EDTA tubes. Then, Ficoll density gradient centrifugation was used to isolate peripheral blood mononuclear cells (PBMCs) for analysis by flow cytometry. Plasma samples were collected after centrifugation and were frozen $\left(\right.$ at $\left.-80^{\circ} \mathrm{C}\right)$ prior to using the cytometric bead array (CBA; kit no. 11363D, Dynal, Invitrogen, USA) for isolation of $\mathrm{CD} 4^{+} \mathrm{CD} 25^{-} \mathrm{T}$ cells). Cell purity was based on a threshold of $97 \%$ from flow cytometry. Cell activity (determined using the trypan blue exclusion assay) was based on a threshold of $95 \%$.

\subsection{Extraction of total RNA extraction and synthesis of cDNA}

Total RNA (including miRNAs) samples were isolated from $\mathrm{CD} 4{ }^{+} \mathrm{CD} 25^{-} \mathrm{T}$ cells using the miRNeasy Mini Kit (Qiagen, Germany) according to the manufacturer's instructions. Following confirmation of purity (average $\mathrm{OD}_{260 \mathrm{~nm}} / \mathrm{OD}_{280 \mathrm{~nm}}=1.98$ ), cDNA was synthesized using oligo-dT primers and RevertAid ${ }^{\mathrm{TM}} \mathrm{H}$ Minus reverse transcriptase (Fermentas, Lithuania). The miScript II RT kit (Qiagen) was used for synthesis of miRNA cDNA. Negative controls (no first-strand synthesis) were synthesized using reverse transcription without reverse transcriptase.

\subsection{LightCycler real-time PCR}


The cDNA levels of IL-4 were determined using real-time PCR with the Quantitect ${ }^{\mathrm{TM}}$ SYBR green PCR Kit (Takara, Japan) and a LightCycler ${ }^{\circledR} 2.0$ (Roche Molecular Biochemicals, Switzerland). The primers were: 5'-TCATTTTCCCTCGGTTTCAG-3' (forward) and 5'ATAGGTGTCGATTTGCAGTG-3' (reverse). Real-time PCR with the miScript SYBR Green PCR Kit (Qiagen) and the LightCycler® 2.0 were used to quantitate the cDNA levels of miR-24 and miR-27. The primers were synthesized from recommendations in the miRNA miScript Primer Assay (Qiagen). The second derivative maximum method was used to identify the crossing point $(\mathrm{Cp})$ with LightCycler software version 3.5.30 (Roche Molecular Biochemicals). After normalization using Relative Quantification Software version 1.0 (Roche Molecular Biochemicals), the levels of the PCR products were presented relative to those of GAPDH (target genes) or U6 (miRNAs).

\subsection{Flow cytometry analysis of Th2 cells}

To measure expression of markers in the cytoplasm of Th2 cells, these cells were cultured in a $37^{\circ} \mathrm{C} / 5 \% \mathrm{CO}_{2}$ incubator ionomycin (250 ng/mL; SigmaAldrich), PMA (25 ng/mL; Sigma-Aldrich), and monensin (20 ng/mL; eBio-science, San Diego, CA, USA) were added as stimulation for $24 \mathrm{~h}$. Then, cells were stained for different markers, and they were fixed and permeabilized using the Cytofix/Cytoperm kit (eBioscience). The cells were stained with specific antibodies (or corresponding isotype-matched controls) and then analyzed using a FACS Canto II flow cytometer (BD Biosciences, Mississauga, ON, Canada). Staining with the fixable viability stain 
450 (FVS450, BD Biosciences) was used to determine viability. Validated antibodies (CD3-eFour450A, CD8-FITC, and IL-4-PE) were purchased from eBioscience.

\subsection{CBA detection of plasma IgE, IL-4 and IL-13}

A CBA kit (e Bioscience) was used to measure the plasma levels of IgE, IL-4, and IL-13. Each sample was measured twice.

\subsection{Cell transfection and culture}

The roles of miR-24 and miR-27 in Th2 cells were examined using transfection experiments in PBMCs. First, PBMCs were isolated from healthy controls or patients using Ficoll density gradient centrifugation, and then mimics (Ctrl-m, miR-24-m, or miR-27-m) or inhibitors (Ctrl-i, miR-24-i, or miR-27-i) from RiboBio (Guangzhou, China) were transfected into the PBMCs using the riboFECT CP Transfection Kit (Guangzhou, China). Cells were cultured in RPMI-1640 medium (Gibco, CA, USA) that was supplemented with $15 \%$ fetal calf serum (Gibco, CA, USA) and maintained at $37^{\circ} \mathrm{C} / 5 \% \mathrm{CO}_{2}$ in 24 -well plates $\left(3 \times 10^{6}\right.$ cells per $\left.\mathrm{mL}\right)$. Cells were then harvested for flow cytometry and RT-PCR analyses.

\subsection{Statistical analysis}

Statistical analyses were performed using SPSS software for Windows version 13.0 (SPSS Inc., USA). Data are expressed as means \pm standard deviations. For comparisons of multiple groups, a one-way analysis of variance was used. For comparison of two groups, Student's $t$-test was used. $P$-values below 0.05 were considered significant. 


\subsection{Results}

\subsection{Patients with atopic and non-atopic INS have increased serum IgE levels}

We first measured the plasma levels of $\operatorname{IgE}$ in the five different groups using a CBA (Fig. 1). Analysis of IgE concentration indicated significantly greater levels in the AA group $(1350.67 \pm 837.39 \mathrm{IU} / \mathrm{mL}, P<0.05)$ and the ANA group $(441.01 \pm$ $357.45 \mathrm{IU} / \mathrm{mL}, P<0.05)$ than in the $\mathrm{Ctrl}$ group $(57.76 \pm 48.25 \mathrm{IU} / \mathrm{mL})$. In addition, the AA group had higher levels of IgE than other groups (all $P<0.05$ ). Compared with the ANA group, the Ctrl, ReNA, and ReA groups had significantly reduced levels of $\operatorname{IgE}$ (all $P<0.05$ ). However, the ReA and ReNA groups had no significant difference $(P>0.05)$.

\subsection{Patients with active phase INS have over-expression of Th2 cells and associated} cytokines

We quantified the Tregs from whole blood samples in the five groups using flow cytometry (Fig. 2A, B). The percentage of peripheral Th2 cells in the AA group $(5.60 \% \pm 1.21)$ and the ANA group $(4.23 \% \pm 0.92)$ were significantly greater than in the Ctrl group $(3.29 \% \pm 1.02$, both $P<0.05)$. As with $\mathrm{IgE}$, there was no significant difference between the ReA and ReNA groups $(P>0.05)$. Also as with IgE, the AA group had a higher percentage of Th2 cells than all other groups (all $P<0.05$ ). Compared with the ANA group, the Ctrl and the ReNA groups had significantly reduced percentages of Th2 cells (both $P<0.05$ ), but the percentages were similar in the ANA and ReA groups $(P>0.05)$. 
We then measured the plasma levels of IL-4 and IL-13 in all five groups using CBA (Fig. 2C, D). Relative to the control group, The AA group had higher levels of IL-4 and IL-13 (IL-4: 66.66 pg/mL \pm 43.85 vs. 23.29 pg/mL $\pm 13.91, P<0.05$; IL-13: $65.15 \mathrm{pg} / \mathrm{mL} \pm 16.50$ vs. $15.66 \mathrm{pg} / \mathrm{mL} \pm 8.16, P<0.05)$ and in the ANA group (IL-4: $37.04 \mathrm{pg} / \mathrm{mL} \pm 17.39$ vs. $23.29 \mathrm{pg} / \mathrm{mL} \pm 13.91, P<0.05 ; \mathrm{IL}-13: 33.15 \mathrm{pg} / \mathrm{mL} \pm 14.09$ vs. $15.66 \mathrm{pg} / \mathrm{mL} \pm 8.16, P<0.05)$. However, there was no significant difference between the ReA and ReNA groups in these cytokines (both $P>0.05$ ). Compared with the AA group, all other groups had significantly decreased levels of both cytokines (all $P<0.05$ ). Compared with the ANA group, the Ctrl and ReNA groups had significantly reduced levels of these cytokines (both $P<0.05$ ), and the levels were similar in the ANA and RA groups $(P>0.05)$.

\subsection{Patients with non-atopic INS have altered expression of miR-24 and miR-27}

Previous research showed that miR-24 and miR-27 affected Th2 cell expression. Because we identified increased expression of Th2 cells in the ANA group, we wanted to determine whether these miRNAs were also decreased (Figure 3). The results showed that levels of miR-24 and miR-27 were significantly lower in the ANA group than in the Ctrl group (miR-24: $[21.84 \pm 6.86$ vs. $46.03 \pm 10.08] \times 10^{-3}, P<0.05$; miR-27: $[20.72 \pm 4.93$ vs. $\left.37.83 \pm 6.83] \times 10^{-3}, P<0.05\right)$. The levels of these two miRNAs were greater in the ReNA group than in the ANA group, but not up to the levels in the Ctrl group (miR-24: $[35.59 \pm 9.10] \times 10^{-3}, P<0.05$; miR-27: $\left.[32.55 \pm 7.86] \times 10^{-3}, P<0.05\right)$.

\subsection{MiR-24 and miR-27 suppress the expression of Th2 cells and IL-4}


We further examined the relationship between miR-24, miR-27, and Th2 cells by transfection of PBMCs isolated from healthy volunteers and INS patients with ANA using different miRNAs (negative control: Ctrl-m; miR-24 mimic: miR24-m; miR-27 mimic: miR-27-m; miRNA inhibitor negative control: Ctrl-i; miR-24 inhibitor: miRmiR-24-i; miR27 inhibitor: miR-27-i). The results showed that up-regulation of miR-24 (Fig. 4A, B) and miR-27 (Fig. 5A, B) reduced the percentage of Th2 cells (Fig. 4C, D; Fig. 5C, D) and the expression of IL-4 mRNA (Fig. 4E, F; Fig. 5E, F). In agreement, down regulation of miR-24 (Fig. 4G, H) and miR-27 (Fig. 5G, H), increased the percentage of Th2 cells (Fig. 4I, J; Fig. 5I, J) and the expression of IL-4 mRNA (Fig. 4K, L; Fig. 5K, L).

\subsection{Discussion}

A large body of evidence has demonstrated that the immune system may play a crucial role in INS [3], although the pathogenic details of this disease remain mostly unknown. Previous research suggested that INS may be due to an abnormal T cell response or the dysregulation of T lymphocytes [3-8]. MCD is the most common cause of INS [1-2,18-19], and Th2 cells play an important role in many allergic and inflammatory diseases [9-11,25, 29]. More than 60 years ago, Hardwicke et al. reported an association of seasonal proteinuria with pollen sensitivity [12]. Patients with MCD often have allergy-like symptoms, such as bronchial asthma, allergic rhinitis, atopic dermatitis, and urticaria. Some studies also confirmed that patients 
with INS may have increased levels of serum IgE, especially children with INS recurrence $[13-15,18]$. However, the causal relationship between the increased serum level of IgE or atopy and the pathogenesis of INS remains uncertain. We found that children in the active phase of INS with or without atopy had increased plasma levels of IgE compared with healthy controls, and they also had higher levels then children in the remission phase of INS. Among children in remission, the IgE levels of non-atopic children were nearly normal, but the IgE levels of atopic children were high. Thus, children with atopic and non-atopic INS have increased serum levels of $\operatorname{IgE}$. However, further investigations of the underlying cytokine regulation network are needed to more completely understand the relationship between INS and IgE production.

Th2 cells secrete IL-13 and IL-4, and this can promote the production of IgE in B cells [9-11]. In particular, IL-13 induces a change from expression of $\operatorname{IgM}$ to $\operatorname{IgE}$ in $\mathrm{B}$ cells, and also induces podocytes to increase their synthesis of CD80. Increased CD80 expression by podocytes is associated with proteinuria $[13-15,18]$. Elevated levels of IL-13 are present in the urine and serum of patients who have kidney disease, and are also associated with a proteinuria $[13-15,18]$. These previous studies thus suggest that IL-13 functions in the pathogenesis of kidney disease, but the mechanism responsible for an increased IL-13 level is still unclear. Recent studies reported that Th2-related factors, IL-13 and IL-4, have increased levels in patients with active INS [13-15, 18]. Other studies noted that patients with INS have increased proportions of Th2 cells $[8,18,21-22]$, but it was uncertain whether this contributed to the pathogenesis of INS. 
This led us to examine the expression of Th2 cells in peripheral blood and the plasma concentrations of IL-13 and IL-4 in patients with different INS disease states. Our results demonstrated significantly increased levels of Th2 cells, IL-13, and IL-4 in patients with active phase INS with or without atopy. Our results thus suggest that the proteinuria and increased $\operatorname{IgE}$ levels that occur during the active phase of INS, regardless of the presence of atopy, might be due to the increased levels of IL-13 and IL-4, which were caused a drift toward Th2 cells. However, the mechanism responsible for this drift toward Th2 cells needs further study to elucidate the role of the immune system in the pathogenesis of INS.

miRNAs are subtle "master controllers" of gene expression, and function in the pathogenesis of many human diseases, especially chronic and multifactorial diseases [23-24]. Previous studies reported that some specific miRNAs were abnormally expressed in patients with various allergic and autoimmune diseases, such as asthma, systemic lupus erythematosus (SLE), and lupus nephritis, and that their expression also increased with disease activity [25, 30-31 ]. Other specific miRNAs are important for Th2 cell proliferation, differentiation, and immune function [25-27]. In particular, miR-24 and miR-27 suppress allergic inflammation and target a network of regulators of Th2 cell-associated cytokine production [26-27]. However, it is unclear whether changes alterations in the levels of different miRNAs lead to alterations in Th2 expression in patients with active INS. Therefore, we determined the expression of miR-24 and miR-27 and examined their possible role in regulating Th2 cell differentiation. Our results clearly showed that these two miRNAs had low expression 
in pediatric patients with active non-atopic INS. This suggests that the drift toward Th2 cells might be related to the low expression of these MiRNAs in these children.

To verify this hypothesis, we first isolated PBMCs from healthy controls and children with non-atopic INS. Then, we transfected these cells with miR-24 or miR-27 mimics to increase expression, or with miR-24 or miR-27 inhibitors to reduce expression. The results showed that the percentage of Th2 cells and the level of IL-4 decreased when the levels of these miRNAs increased. In agreement, the percentage of Th2 cells and the expression of IL-4 increased when the levels of these miRNAs decreased. These results support our initial hypothesis that miR-24 and miR-27 reduce the percentage of Th2 cells, and suggest they might play an important role in Th2 expression during active INS.

In conclusion, our results indicate that the Th2 related-cytokine IL-13 is related to the pathogenesis of kidney disease, but the mechanisms responsible for the increased level of this cytokine remain unclear. Our results also indicate that pediatric patients with active phase INS that is either atopic or non-atopic have higher levels of IgE, and this might be related to their higher levels of IL-13 and IL-4, which caused by a drift toward Th2 cells. Children with active phase non-atopic INS had reduced levels of miR-24 and miR-27, an increased percentage of Th2 cells, and an increased level of IL-4, and this led to IL-13 over-expression and increased IgE levels. In other words, these two MiRNAs suppress the expression of Th2 cells and play an important role in the drift toward Th2 cells in children with active INS. Therefore, we speculate that these miRNAs should be considered as potential targets for treatment of kidney 
disease. Our research thus provides a new research direction and suggests a possible novel treatment for INS.

\section{Declaration}

\section{Ethical Approval and Coasait to Partidpate}

The study was performed after approval by the Medical Ethics Committee of Shenzhen Children's Hospital, which covered all experimental methods. The parents or legal guardians provided informed consent prior to study enrollment. All methods were performed in accordance with the relevant guidelines and regulations. The research procedures were carried out in line with the proposed guidelines and regulartions(Declaration of Helsinki).

\section{Consent to Publish}

Not applicable

\section{Availability of data and materials}

The dataset that was generated and analvsed in this study is not publicly availoble owing to protect the confidentiality of the respondents, but is available frong the corresponding author on reasonable request.

\section{Competing interests}

The authors declare the they have no conflicts of interests.

\section{Funding}

This study was supported by grants from the Shenzhen Municipal Government of China (no. JCYJ20160429174400950) and the Sanming Project of Medicine in 
Shenzhen (no. SZSM201812002). The authors are also grateful to the patients and healthy volunteers who participated in this study.

\section{Authors' contributions}

Fen-fen Ni,Guang-lei Liu and Shi-lei Jia conceived the sutdy, collected date, analysed data and drafted the manuscript; Ran-ran Chen prepared figures 1-2 and Li-bing Liu prepared figures 3-5. Cheng-rong Li, Jun Yang and Xiao-Jie Gao participated in the design, analysis and helped th draft the manuscript. All the authors read and approved the manuscript.

\section{Acknowledgements}

This study was supported by grants from the Shenzhen Municipal Government of China and the Sanming Project of Medicine in Shenzhen. The authors are also grateful to the patients and healthy volunteers who participated in this study.

\section{Data Availability Statement}

The datasets used and/or analysed during the current study are available from the corresponding author on reasonable request. The raw/processed data required to reproduce these findings cannot be shared at this time as the data also forms part of an ongoing study. 


\section{References}

1. Yang JY, Yao Y. Analysis of 1268 patients with chronic renal failure in childhood: a report from 91 hospitals in China from 1990 to 2002. Zhonghua Er Ke Za Zhi Chinese Journal of Pediatrics. 2004;42:724-30.

2. Vivarelli M , Massella L, Ruggiero B , et al. Minimal Change Disease[J]. Clinical Journal of the American Society of Nephrology, 2016, 12(2):332.

3. Colucci M, Corpetti G, Emma F, et al. Immunology of idiopathic nephrotic syndrome[J]. Pediatric Nephrology, 2017:1-12.

4. Sellier-Leclerc A-L, Duval A, Riveron S et al. A humanized mouse model of idiopathic nephrotic syndrome suggests a pathogenic role for immature cells. Journal of the American Society of Nephrology.. 2007;18:2732-9.

5. Wang L, Li Q, Wang L et al. The Role of Th17/IL-17 in the Pathogenesis of Primary Nephrotic Syndrome in Children. Kidney Blood Press Res. 2013;37:332-45.

6. Fodor P, Saitúa MT, Rodriguez E, González B, Schlesinger L. T-Cell Dysfunction in Minimal-Change Nephrotic Syndrome of Childhood. American journal of diseases of children. 1982;136:713-7.

7. Herrod HG, Stapleton FB, Trouy RL, Roy S. Evaluation of T lymphocyte subpopulations in children with nephrotic syndrome. Clin Exp Immunol. 1983;52:581-5.

8. Kanai T, Shiraishi H, Yamagata $\mathrm{T}$ et al. Th2 cells predominate in idiopathic steroid-sensitive nephrotic syndrome. Clin Exp Nephrol. 2010;14:578.

9. Forsthuber T G . Stability of T-cell lineages in autoimmune diseases[J]. Expert Review of Clinical Immunology, 2012, 8(4):299-301.

10. Tosolini M, Kirilovsky A, Mlecnik B, et al. Clinical Impact of Different Classes of Infiltrating T Cytotoxic and Helper Cells (Th1, Th2, Treg, Th17) in Patients with Colorectal Cancer[J]. Cancer Research, 2011, 71(4):1263-71.

11. Chapoval S, Dasgupta P, Dorsey N J, et al. Regulation of the T helper cell type $2(\mathrm{Th} 2) / \mathrm{T}$ regulatory cell (Treg) balance by IL-4 and STAT6[J]. Journal of Leukocyte Biology, 2010, 87(87):1011-1018.

12. Hardwicke J, Soothill JF, Squire JR, Holti G. Nephrotic syndrome and pollen hypersensitivity. Lancet 1959; I: 499-502. 
13. Mishra O P, Teli A S, Singh U, et al. Serum Immunoglobulin E and Interleukin-13 Levels in Children with Idiopathic Nephrotic Syndrome[J]. Journal of Tropical Pediatrics, 2014, 60(6): 467-471.

14. Hossain A. Serum IgE level in relapsing idiopathic nephrotic syndrome in children[J]. Urology \& Nephrology Open Access Journal, 2019, 7(2).

15. Mishra, O. P . Serum Immunoglobulin E in Idiopathic Nephrotic Syndrome[J]. Journal of Tropical Pediatrics, 2004, 50(3):149-152.

16. Garin E H , Diaz L N, Mu W , et al. Urinary CD80 Excretion Increases in Idiopathic Minimal-Change Disease[J]. Journal of the American Society of Nephrology, 2009, 20(2):260-266.3.

17. Reiser, J. Danger Signaling by Glomerular Podocytes Defines a Novel Function of Inducible B7-1 in the Pathogenesis of Nephrotic Syndrome[J]. Journal of the American Society of Nephrology, 2004, 15(9):2246-2248.

18. Abdel-Hafez M, Shimada M, Lee P Y, et al. Idiopathic Nephrotic Syndrome and Atopy: Is There a Common Link?[J]. American Journal of Kidney Diseases, 2009, 54(5):945-953.

19. Shimada, M., Araya, C., Rivard, C. et al. Minimal change disease: a "two-hit" podocyte immune disorder?[J]. Pediatr Nephrol, 2011, 26(4): 645-649.

20. Carlos, Araya, Leila, et al. T regulatory cell function in idiopathic minimal lesion nephrotic syndrome[J]. Pediatric Nephrology, 2009.

21. Adrogue H E, Borillo J , Torres L, et al. Coincident Activation of Th2 T Cells with Onset of the Disease and Differential Expression of GRO-Gamma in Peripheral Blood Leukocytes in Minimal Change Disease[J]. American Journal of Nephrology, 2007, 27(3):253-261.

22. Carlos E. Araya, Clive H. et al. A case of unfulfilled expectations. Cytokines in idiopathic minimal lesion nephrotic syndrome[J]. Pediatric Nephrology, 21(5):603-610.

23. Dai R, Ahmed S A. MicroRNA, a new paradigm for understanding immunoregulation, inflammation, and autoimmune diseases[J]. Translational Research, 2011, 157(4): 163-179.

24. Pauley K M, Cha S, Chan E K L. MicroRNA in autoimmunity and autoimmune diseases[J]. Journal of autoimmunity, 2009, 32(3): 189-194..

25. Simpson L J, Patel S, Bhakta N R, et al. A microRNA upregulated in asthma airway T cells promotes TH2 cytokine production[J]. Nature Immunology, 2014, 15(12):1162-70.

26. Pua H H, Steiner D F, Patel S, et al. MicroRNAs 24 and 27 suppress allergic inflammation and target a network of regulators of T helper-2 cell-associated cytokine production[J]. Immunity, 
2016, 44(4):821-832.

27. Sunglim Cho, ChengJang Wu, Tomoharu Yasuda, et al. miR-23 27 24 clusters control effector $\mathrm{T}$ cell differentiation and function[J]. Journal of Experimental Medicine, 2016, 213(2):jem.20150990.

28. Nephrology Group, Pediatrics Branch of Chinese Medical Association. Evidence-based Guidelines for Diagnosis and Treatment of Common Kidney Diseases in Children (1): Evidence-based Guidelines for Diagnosis and Treatment of Hormone Sensitivity, Relapse/Dependent Nephrotic Syndrome (Trial) [J]. Chinese Journal of Pediatrics, 2009, 47 (3): 167-170.

29. NakayamaToshinori, HiraharaKiyoshi, OnoderaAtsushi, et al. Th2 Cells in Health and Disease[J]. Annual Review of Immunology, 2017, 35(1):53-84.

30. Mohsen H , Paulina K , Ekaterina V R , et al. The Involvement of MicroRNAs in Modulation of Innate and Adaptive Immunity in Systemic Lupus Erythematosus and Lupus Nephritis[J]. Journal of Immunology Research, 2018, 2018:1-15.

31. Stypińska Barbara, Agnieszka P G . Cytokines and MicroRNAs as Candidate Biomarkers for Systemic Lupus Erythematosus[J]. International Journal of Molecular Sciences, 2015, 16(10):24194-24218. 


\section{Figure Legends}

Fig. 1. Children with atopic and non-atopic INS have increased serum levels of IgE. Here and below: all data are shown as means \pm SDs; ${ }^{*} P<0.05$ and ${ }^{*} P>0.05$; and abbreviations are healthy control $(\mathrm{Ctrl}, \mathrm{n}=20)$, active phase atopic $(\mathrm{AA}, \mathrm{n}=6)$, active phase non-atopic (ANA, $n=14)$, remission phase atopic $(\operatorname{ReA}, n=6)$, remission phase non-atopic (ReNA, $\mathrm{n}=14)$.

Fig. 2. Children with INS have increased levels of Th2 cells and Th2-related

cytokines. A. Flow cytometric analysis of Th2 cells. B. Percentages of Th2 cells. C. Plasma concentrations of IL-4. D. Plasma concentrations of IL-13.

Fig. 3. Children with INS have reduced expression of miR-24 and miR-27 in CD4 $^{+} \mathbf{C D 2 5}^{-} \mathbf{T}$ cells. Expression of miRNAs (relative to U6) were determined by real-time PCR.

Fig. 4. MiR-24 down-regulates the expression of Th2 cells and Il-4. Here and in Fig. 5: expression of miRNAs (relative to U6) and IL-4 (relative to GAPDH) were determined by real-time PCR; ANA $(n=6)$, Crtl $(n=6)$; and abbreviations are Ctrl-m (miRNA negative control), miR-24-m/miR-27-m (miR-24/miR-27 mimic), Ctrl-i 
(miRNA inhibitor negative control), miR-24-i/miR-27-i (miR-24/miR-27 inhibitor).

Fig. 5. MiR-27 down-regulates the expression of Th2 cells and IL-4. 
Figures

\section{Fig.1}

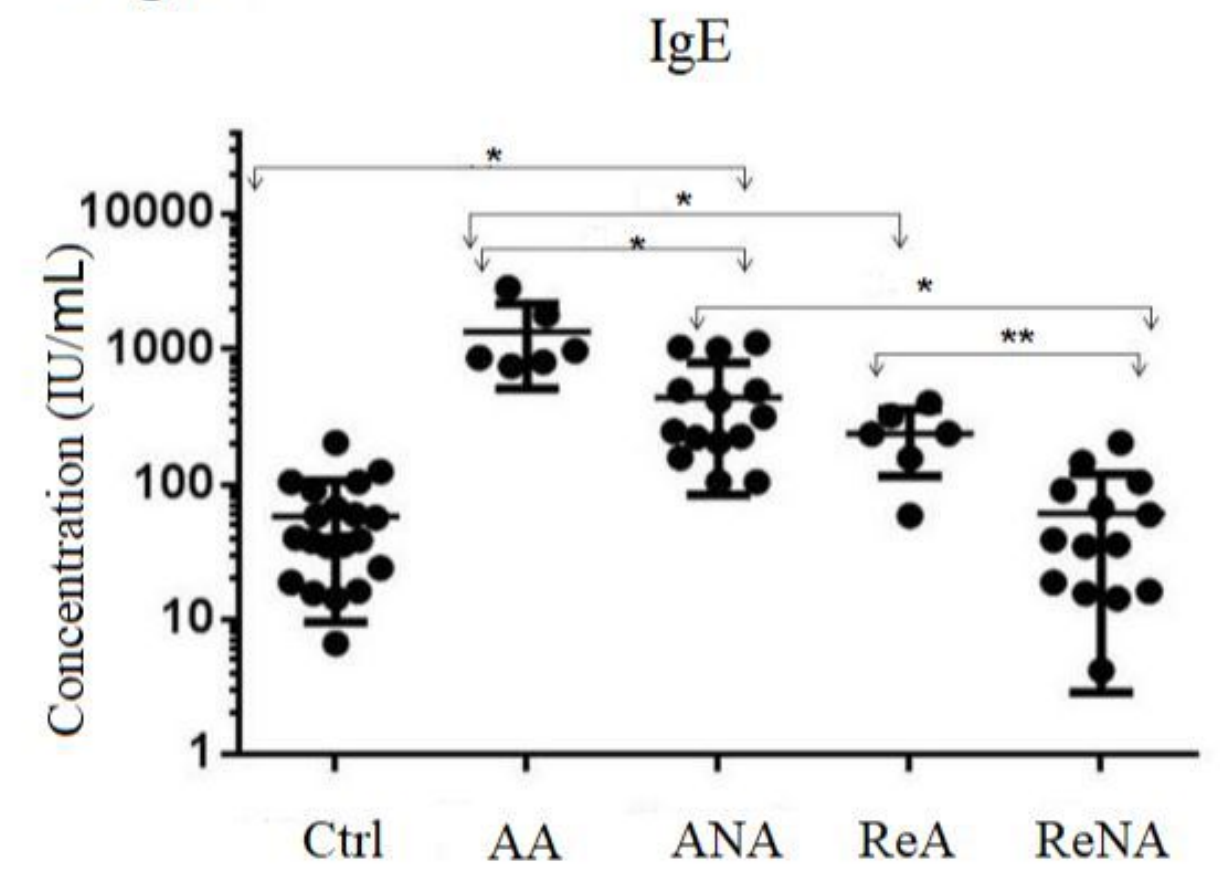

Figure 1

Children with atopic and non-atopic INS have increased serum levels of IgE. Here and below: all data are shown as means $\pm S D s ; * P<0.05$ and $* * P>0.05$; and abbreviations are healthy control $(C t r l, n=20)$, active phase atopic (AA, $n=6)$, active phase non-atopic (ANA, $n=14)$, remission phase atopic $(\operatorname{ReA}, n=$ $6)$, remission phase non-atopic (ReNA, $n=14)$. 
Fig.2

A.
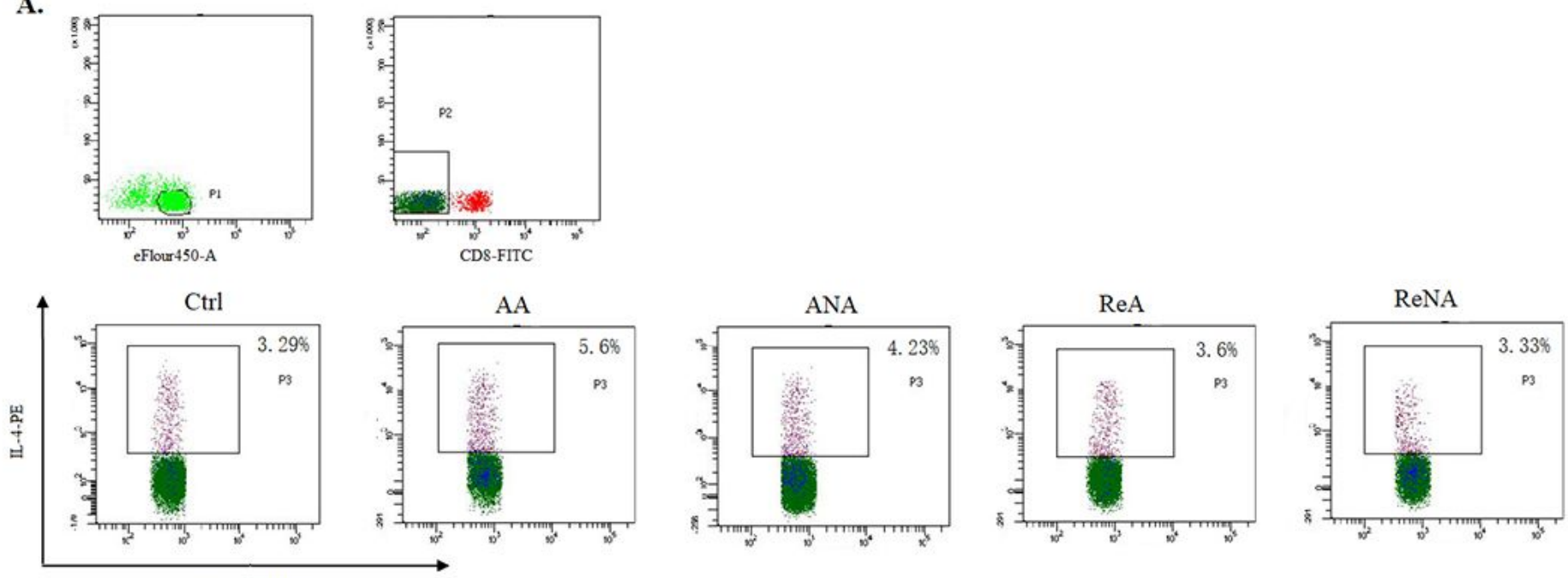

B.

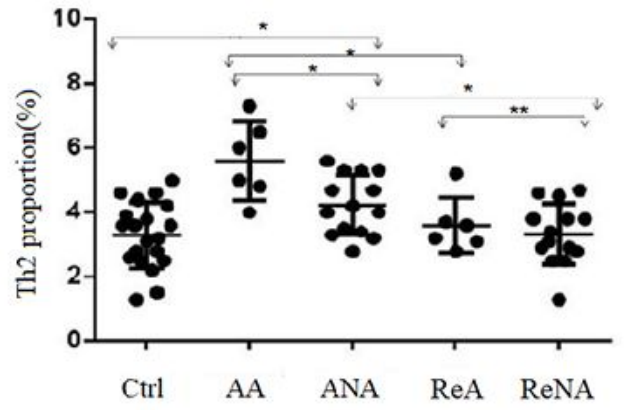

C.

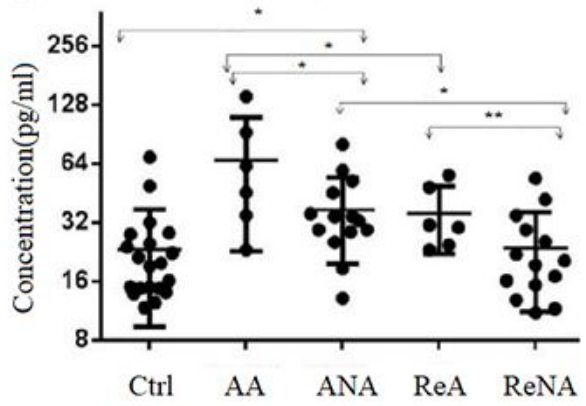

D.

IL-13

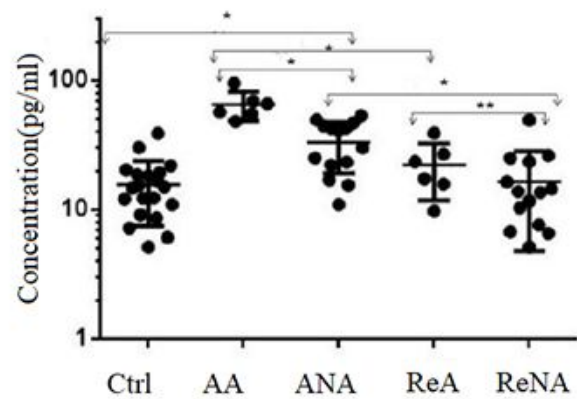

Figure 2

Children with INS have increased levels of Th2 cells and Th2-related cytokines. A. Flow cytometric analysis of Th2 cells. B. Percentages of Th2 cells. C. Plasma concentrations of IL-4. D. Plasma concentrations of IL-13. 
Fig.3

A.

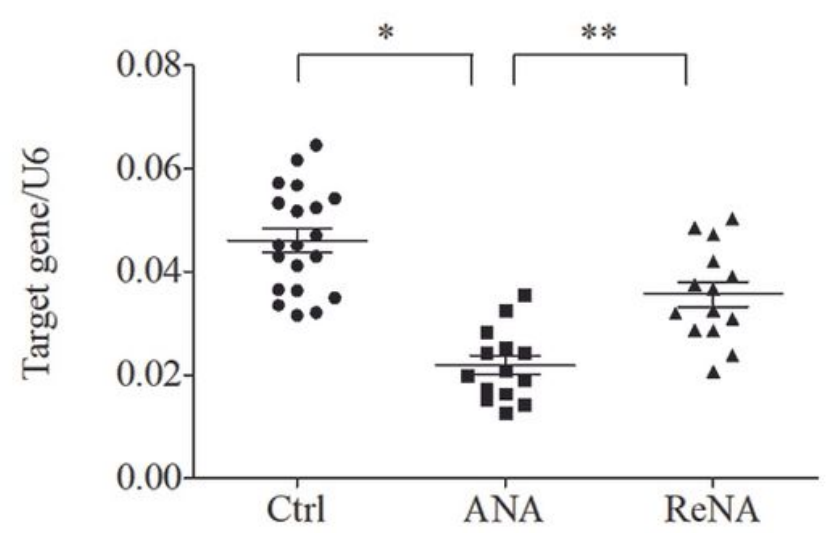

B.

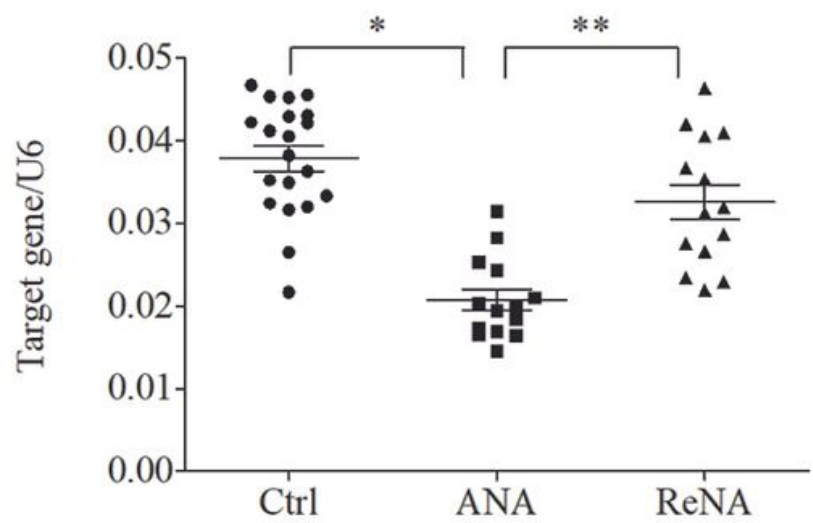

Figure 3

Children with INS have reduced expression of miR-24 and miR-27 in CD4+CD25- T cells. Expression of miRNAs (relative to U6) were determined by real-time PCR. 


\section{Fig.4}

A.

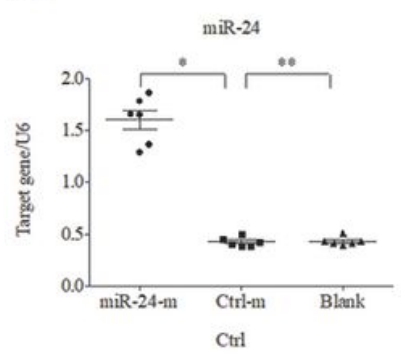

C.

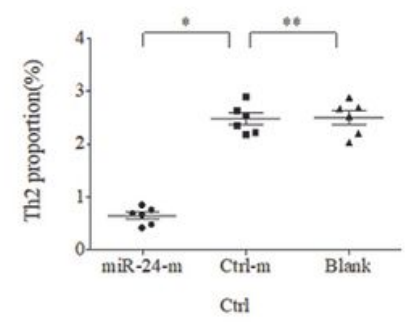

E.

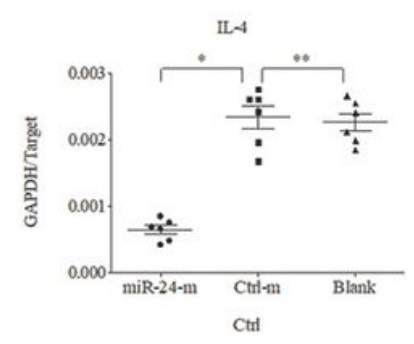

B.

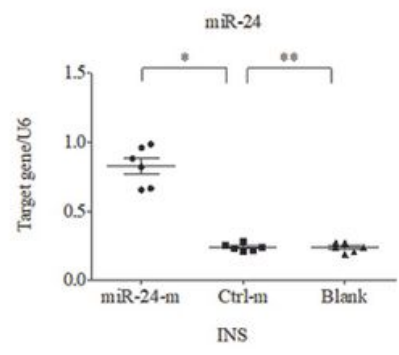

D.

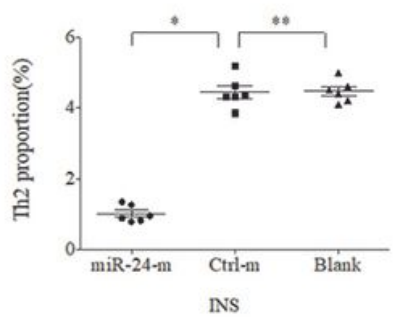

F.

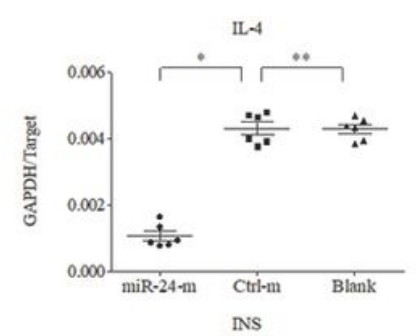

G.

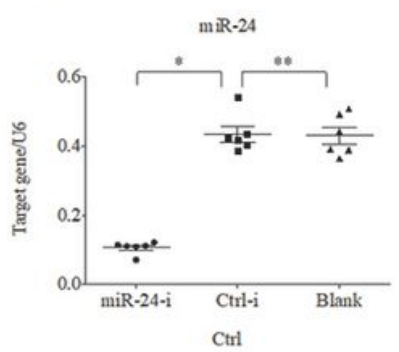

I.

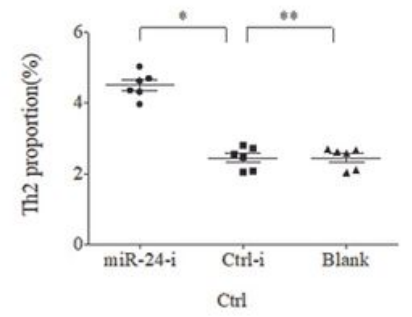

K.

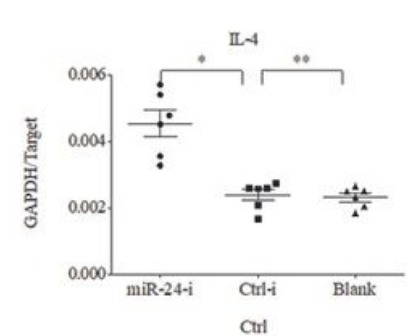

H.

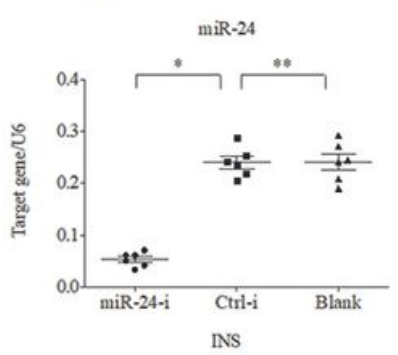

J.

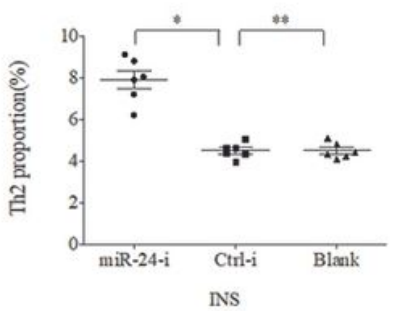

L.

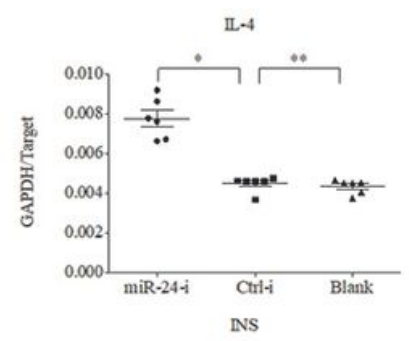

Figure 4

MiR-24 down-regulates the expression of Th2 cells and II-4. Here and in Fig. 5: expression of miRNAs (relative to U6) and IL-4 (relative to GAPDH) were determined by real-time PCR; ANA ( $n=6)$, Crtl $(n=6)$; and abbreviations are Ctrl-m (miRNA negative control), miR-24-m/miR-27-m (miR-24/miR-27 mimic), Ctrl-i (miRNA inhibitor negative control), miR-24-i/miR-27-i (miR-24/miR-27 inhibitor). 
Fig.5
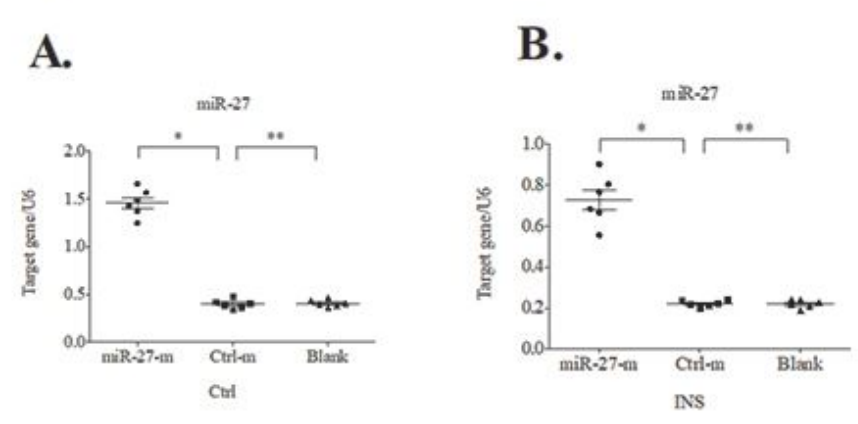

G.

H.
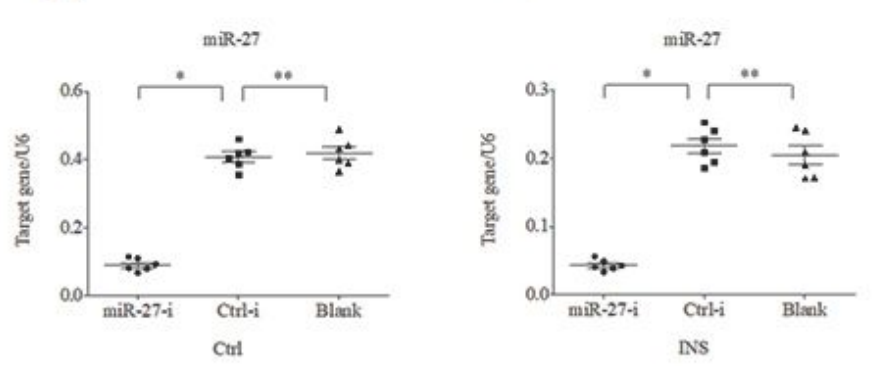

C.

D.

I.

$\mathbf{J}$
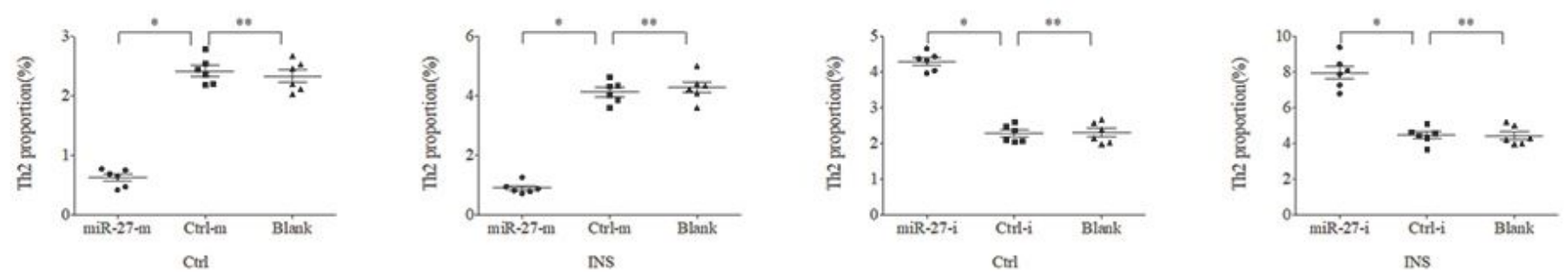

E.

F.

K.

L.

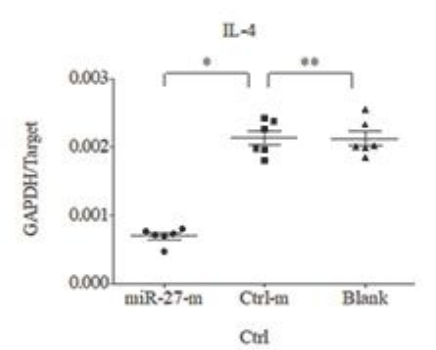

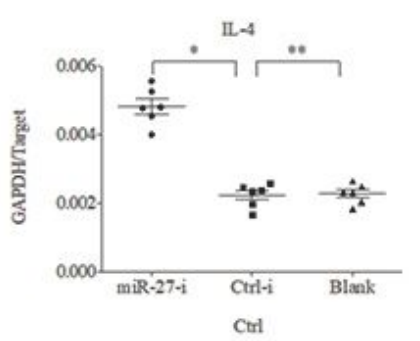

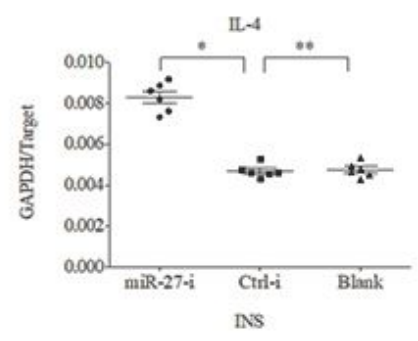

\section{Figure 5}

MiR-27 down-regulates the expression of Th2 cells and IL-4. 